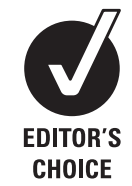

CHOICE

\title{
Utilising improvement science methods to optimise medication reconciliation
}

\author{
Christine M White, ${ }^{1}$ Pamela J Schoettker, ${ }^{2}$ Patrick H Conway, ${ }^{1,2}$ Maria Geiser, ${ }^{2}$ \\ Jason Olivea, ${ }^{2}$ Raymond Pruett, ${ }^{2}$ Uma R Kotagal ${ }^{2}$
}

\begin{abstract}
${ }^{1}$ Division of General and Community Pediatrics, Cincinnati Children's Hospital Medical Center, Cincinnati, Ohio, USA

2James M Anderson Center for Health Systems Excellence, Cincinnati Children's Hospital Medical Center, Cincinnati, Ohio, USA

Correspondence to Dr Christine White, Division of General and Community Pediatrics, Cincinnati Children's Hospital Medical Center, 3333 Burnet Avenue, Cincinnati, OH 45229-3039, USA;

christine.white@cchmc.org
\end{abstract}

Accepted 9 January 2011 Published Online First 11 February 2011

\section{ABSTRACT}

Background: In 2005, The Joint Commission included medication reconciliation as a National Patient Safety Goal to reduce medication errors related to omissions, duplications and interactions. Hospitals continue to struggle to implement successful programmes that meet these objectives.

Methods: The authors used improvement methods and reliability principles to develop and implement a process for medication reconciliation completion at admission at a large, paediatric medical centre. Medication reconciliation was defined as recording a complete and accurate list of each patient's medications within 20 min of admission by the nurse and reconciliation of those medications within $24 \mathrm{~h}$ of admission by the physician. Interventions focused on five main areas: leadership and support from senior physicians and nurses to sustain a culture of safety; simplification and standardisation of the electronic medication reconciliation application; clarifying roles and responsibilities; creating a highly reliable and visible system; and sustainability.

Results: At baseline, only $62 \%$ of patients had their medications reconciled within $24 \mathrm{~h}$ of admission. Over a 9-month period, $\geq 90 \%$ medication reconciliation was achieved within $24 \mathrm{~h}$ of admission. These results have been sustained for 27 months.

Conclusions: Through the use of improvement methods and reliability science, a sustainable process for medical reconciliation completion at admission was successfully achieved at a large, busy academic children's hospital.

Medication errors and preventable adverse drug events (ADEs) in healthcare facilities continue to be serious problems. In response to mounting safety concerns, in 2004, The Joint Commission (a USA-based, notfor-profit organisation that accredits and ertifies healthcare organisations) announced National Patient Safety Goal 8, calling for healthcare organisations to 'accurately and completely reconcile medications across the continuum of care. ${ }^{1}$ Accredited organisa- tions were required to develop and test processes for medication reconciliation to be implemented by January 2006. Medication reconciliation is a formal process of obtaining a complete and accurate list of each patient's current home prescription and non-prescription medications-including name, dosage, frequency and route-and comparing hospital admission, transfer and discharge medication orders to that list. ${ }^{2}$

Since its introduction, many organisations have struggled to develop effective and efficient processes to meet Safety Goal 8. A 2006 survey of hospitalists found that medication reconciliation was fully implemented at only $48 \%$ of respondent's hospitals. ${ }^{3}$ In a 2009 survey, $57 \%$ of hospital pharmacy directors reported that their hospital had a medication reconciliation process that worked well. ${ }^{4}$

Cincinnati Children's Hospital Medical Center (CCHMC) had also struggled with meeting the Goal 8 mandate. Even after adding an electronic reconciliation application, only about $62 \%$ of patients had their medications reconciled at admission. As a result, an executive steering committee was formed to understand the problem, and an improvement team was launched to implement solutions.

This article describes the use of improvement methods ${ }^{5}$ and reliability science ${ }^{6}$ to develop and implement a sustained medication reconciliation process to improve patient safety and compliance with Safety Goal 8 .

\section{METHODS}

\section{Setting}

CCHMC is a large, urban paediatric academic medical centre located in the Midwest USA. In fiscal year 2009, CCHMC had over 31000 inpatient admissions in 511 registered inpatient beds. Over 2300 health professional 
students, residents and fellows receive clinical and research training annually. In 2002, CCHMC implemented a system to integrate clinical information that included computerised clinical order entry, clinical documentation and an electronic medication administration record.

\section{Planning the intervention}

The improvement team, consisting of physician and nursing leaders, frontline nurses and physicians, a quality-improvement consultant, a data analyst, and a patient safety project manager, mapped the existing reconciliation process (figure $1 \mathrm{~A}$ ), conducted a failure mode and effects analysis, ${ }^{7} 8$ and examined the key drivers that prioritised interventions. The key drivers were revised over time. Figure 2 depicts the final, highly accurate key driver diagram. Our specific aim was to increase and sustain completion of medication reconciliation at admission to greater than $90 \%$ for inpatient medical services. Reconciliation was defined as recording a complete and accurate list of each patient's medications within $20 \mathrm{~min}$ of admission by the nurse and reconciliation of those medications within $24 \mathrm{~h}$ of admission by the physician.

\section{Improvement activities}

CCHMC uses The Model for Improvement ${ }^{5}$ as our framework for developing and testing rapid changes that will lead to improvement. The model has four key elements: aim, measurement, ideas for change and tests of change. Interventions focused on five main areas. Guided by quality-improvement consultants, changes were tested through a series of Plan-Do-Study-Act (PDSA) cycles. ${ }^{5}$ Strategies were initially tested on a paediatric medical/surgical unit and later spread gradually to other medical services.

1. Leadership and support to sustain a culture of safety

- Process owners and senior leaders worked to remove barriers to progress, such as lack of alignment of units and paediatric residents with this institutional goal.

- The Chief of Staff confirmed the project as an institutional priority to unit leaders, holding operational personnel accountable for their performance.

- A paediatric chief resident was added to the improvement team. All of the chief residents investigated daily failures to reconcile medications and communicated results to residents, the primary prescribers, holding them individually accountable. The prioritisation was further supported by the paediatric residency program director.

- Medication reconciliation completion constituted a small piece of a hospital-wide initiative launched by CCHMC in 2006 to become a high-reliability organisation $^{6}$ and decrease serious safety events.

- Since 2005, all patient care providers have been asked to voluntarily and anonymously complete the
Agency for Healthcare Research and Quality Hospital Survey on Patient Safety Culture. ${ }^{9}$

2. Simplification and standardisation of the electronic medication reconciliation application

- Streamlined the workflow for all nurses and prescribers to decrease variation in process completion (figure 1B).

- To ensure a more readily available, updated medication reconciliation for physicians to verify early in the admission process, medication reconciliation was moved from the 20th item completed by the nurse while taking an admission history to the 3rd item completed.

- Internal development of an electronic medication reconciliation program with:

a. Separate views and functions for nurses (create/ update medication list) and prescribers (reconcile medication list).

b. Patient information interfaced with the hospital registration system.

c. Individual fields with drop-down lists and autocompletion features for dose, frequency, route, time of last dose and additional comments (figure 3).

d. Medication libraries containing trade and generic drug information within the application.

e. Simple reconciliation functions such as hold, continue or discontinue.

f. Access to prior medication lists for repeat patient encounters.

- Improved error identification: large red boxes were placed around data fields with missing or incomplete required information, making these fields easier to find.

- System integration: the application was integrated with the primary electronic medical record used for order entry. A prompt with a quick link to the medication reconciliation form was placed at the beginning of the order-entry pathway and remained visible until medications were reconciled (figure 4).

- Integration with discharge summary: medication information from disparate electronic databases was compiled into the discharge summary system. Medications from the reconciliation application and those administered during the previous $48 \mathrm{~h}$ were viewable, allowing the physician to continue, discontinue or revise medications. An updated list was included with discharge documentation provided to families.

3. Clarifying roles and responsibilities

- Two weeks prior to the initiation of the project on a unit, an introductory meeting led by the project manager was held with the resident team and nursing and physician leaders of the unit. A Microsoft PowerPoint presentation described the enhancements of the medication reconciliation program and expectations for its completion.

- An algorithm was developed to sustain compliance with reconciliation expectations (figure 5). 


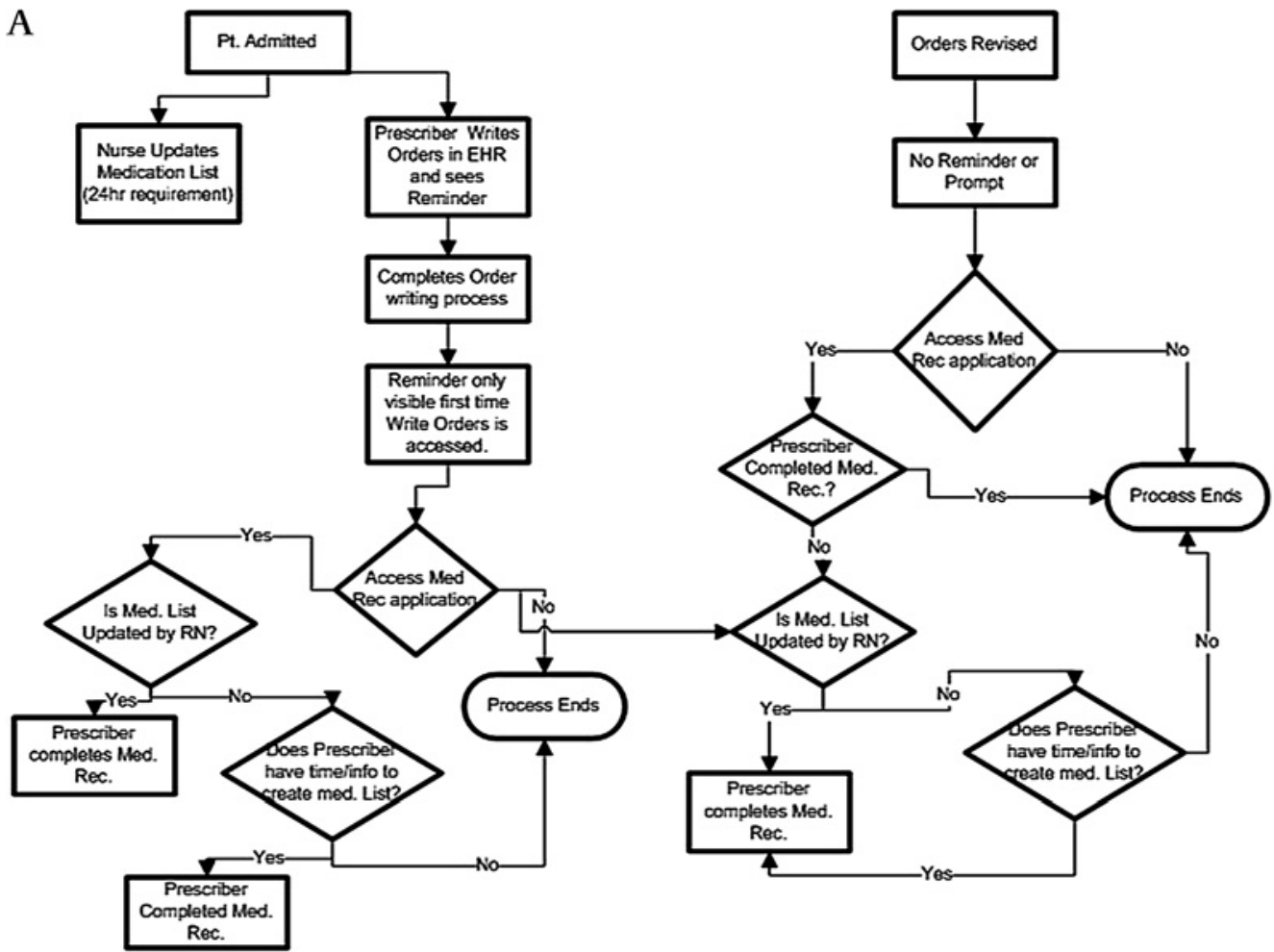

B

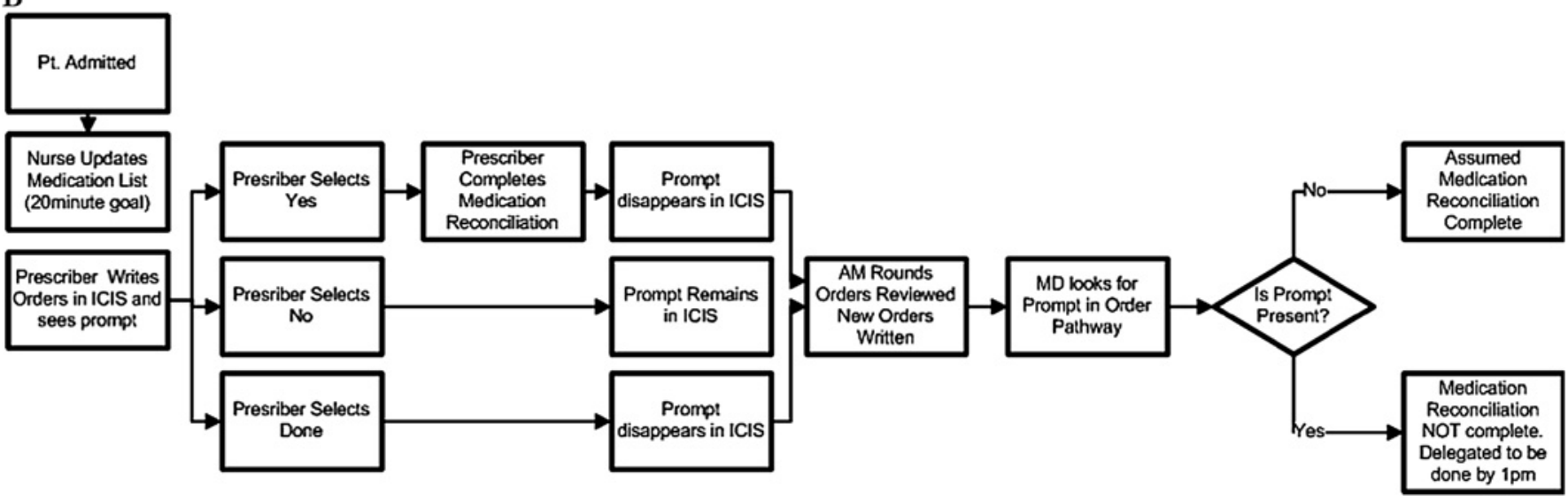

Figure 1 Original and revised medication reconciliation flow diagrams. (A) Original medication reconciliation process.

(B) Revised workflow for medication reconciliation completion. EHR, electronic health record; ICIS, integrating clinical information system (the HER); MD, medical doctor; Med Rec, medication reconciliation; Pt, patient; RN, registered nurse.

- Each inpatient unit identified physician and nursing directors as process owners responsible for monitoring unit performance and responding to failures. The chief residents served as process owners responsible for medication reconciliation by residents. One chief resident was designated primary owner. They educated each resident team about the expectations of medical reconciliation at monthly team meetings, assured that each resident, including outside rotators, had access to the reconciliation programme and were responsible for identifying and mitigating failures. They received weekly updates on failures and had targeted, specific discussions with residents to identify system and individual performance-failure modes. System errors (eg, failure of the electronic medical record prompt to appear) were addressed and resolved, and educational gaps were explored. For example, initially, residents did not realise that they should complete medical reconciliation, even if the patient did not have any home medications.

- Reminders to complete medication reconciliation were posted on wheeled computers used during rounds. Outstanding reconciliations were addressed during rounds with the expectation to to complete them then or to place them on the team's daily 'to do' list.

4. Creating a highly reliable and visible system

- An automated data-reporting system allowed process owners to monitor admissions and evaluate 
Figure 2 Cincinnati Children's Hospital Medical Center (CCHMC) inpatient medical services medication reconciliation key driver diagram.

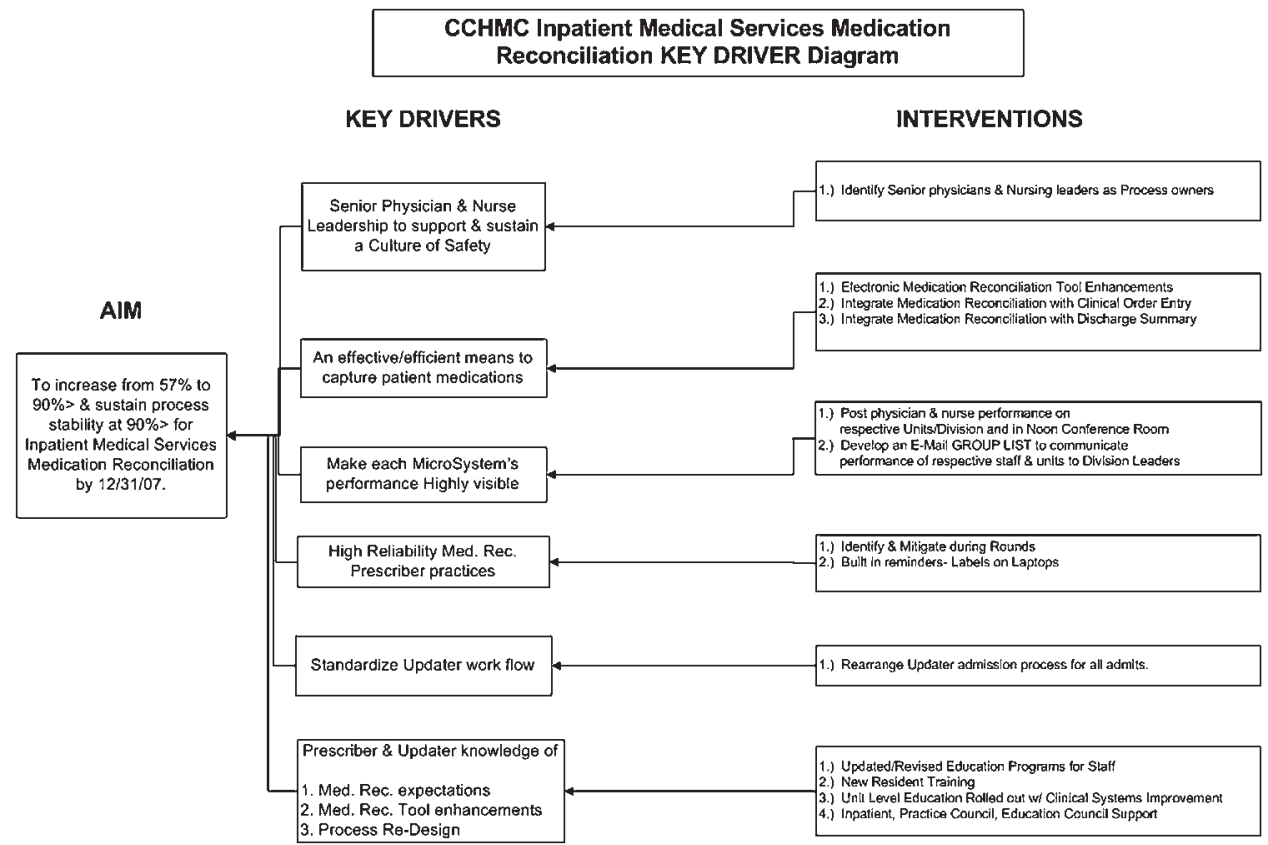

the performance of units and resident teams weekly.

- Data on compliance for each service and unit were reviewed by the project manager weekly, sent to the chief residents and posted on a large scorecard in the resident conference room (figure 6). Current compliance for each team was highlighted at monthly conferences.

- Compliance was included on strategic scorecards ${ }^{10} 11$ to allow senior leadership to monitor the performance of each unit individually, as well as to compare each unit's performance

5. Sustainability

- At the end of each academic year, departing chief residents met with the incoming chiefs to relay the responsibilities of the position and lessons learnt.
A new scorecard was placed in the resident conference room every 6 months.

- Process owners, unit and hospital-wide leaders, received weekly email updates on medication reconciliation, including data on overall compliance and links to performance breakdowns. Some units posted their individual compliance in locations visible to staff and patients.

- Units presented their results quarterly to a team responsible for hospital-wide clinical systems improvement.

- All employees could view performance control charts on the hospital intranet.

- Annual training of new resident physicians and fellows completed by a project manager for patient safety in the spring of each year included a review
Figure 3 Screen shot of prescriber view of medication reconciliation program.

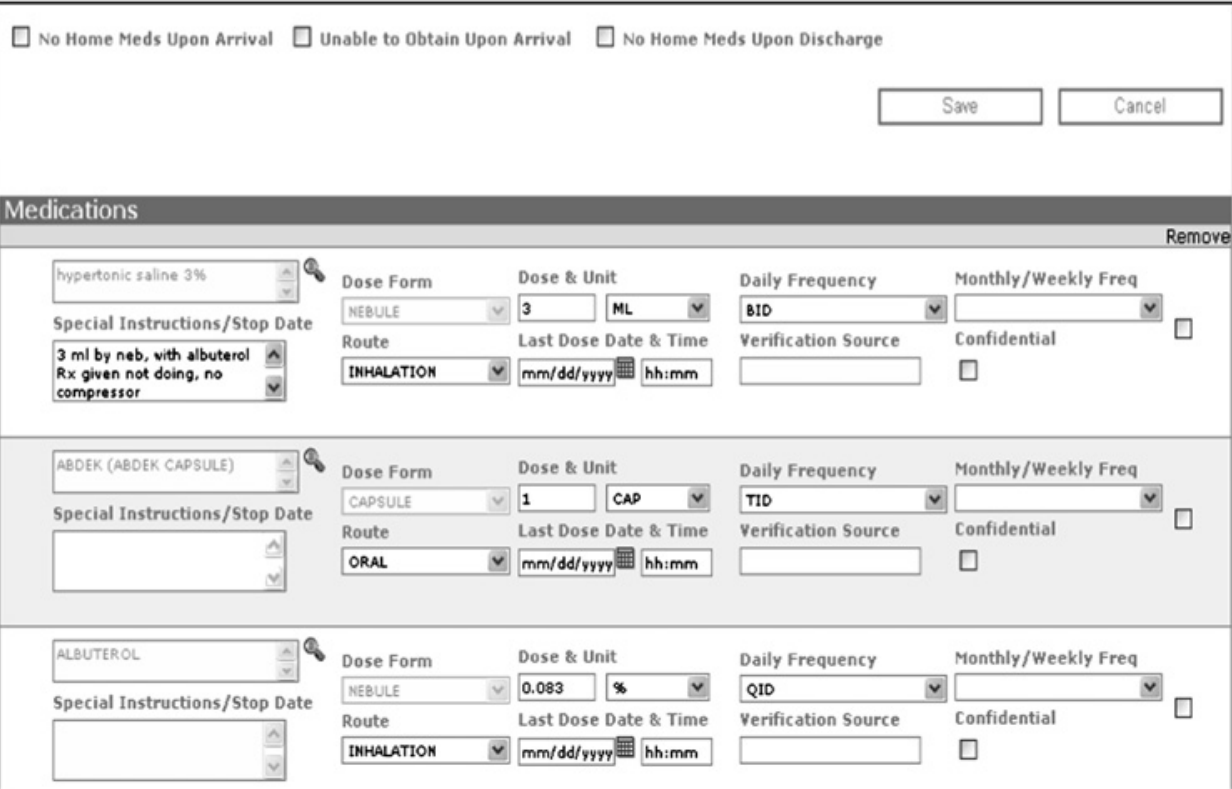




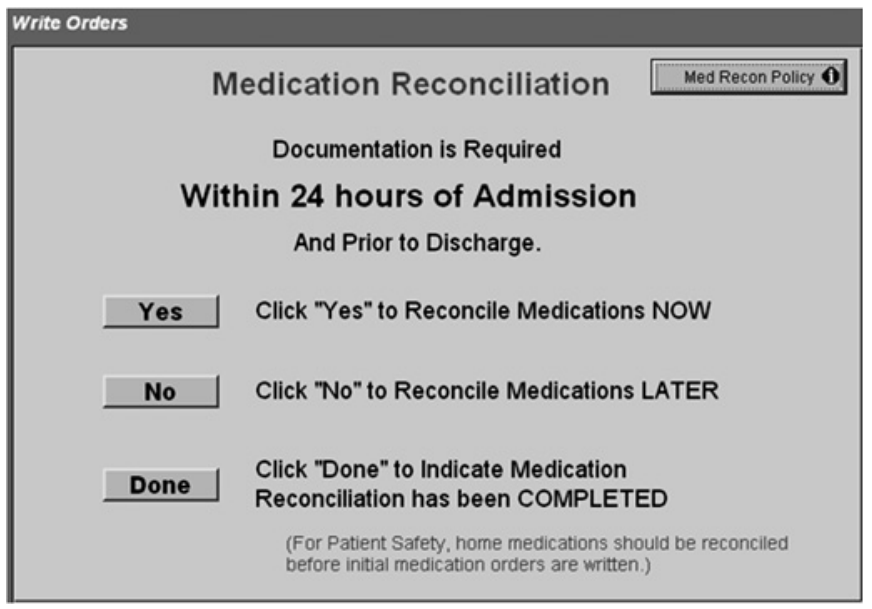

Figure 4 Reminder prompt appearing in the electronic medical record.

of the medication reconciliation application and the expectations of completion within $24 \mathrm{~h}$ of admission.

- Training for new nurses was incorporated into their standard orientation.

\section{Measures}

The primary outcome measure was the percentage of medical patients with medication reconciliation completed within $24 \mathrm{~h}$ of admission.

\section{Data collection}

Data were obtained weekly from automated reports merging admitting and registration information from the primary electronic medical record with data from the electronic medication reconciliation application. Two separate reports were run daily. All admissions within a $24 \mathrm{~h}$ period were extracted from the admission/ discharge/transfer electronic system. A report on the status of the medication reconciliation for each patient within $48 \mathrm{~h}$ of admission was obtained from the electronic medication reconciliation system. Medication reconciliation status was categorised as (1) reconciled, or compliant with process, or (2) not reconciled, non-compliant with process. The report did not contain the status of the reminder prompt within the order-writing system, as the prompt was a built-in reminder and convenience link to streamline workflow. The Microsoft Access database was monitored daily by a project manager.

\section{Analysis}

Data on completed medication reconciliations at admission were plotted on a statistical process control chart to examine the impact of interventions over time. Control charts are commonly used in industry to analyse process-improvement efforts and identify common-cause variation (variation caused inherently by a system) and
Figure 5 Accountability algorithm.
Inpatient Medical Services Admit Medication Reconciliation Compliance Process Map

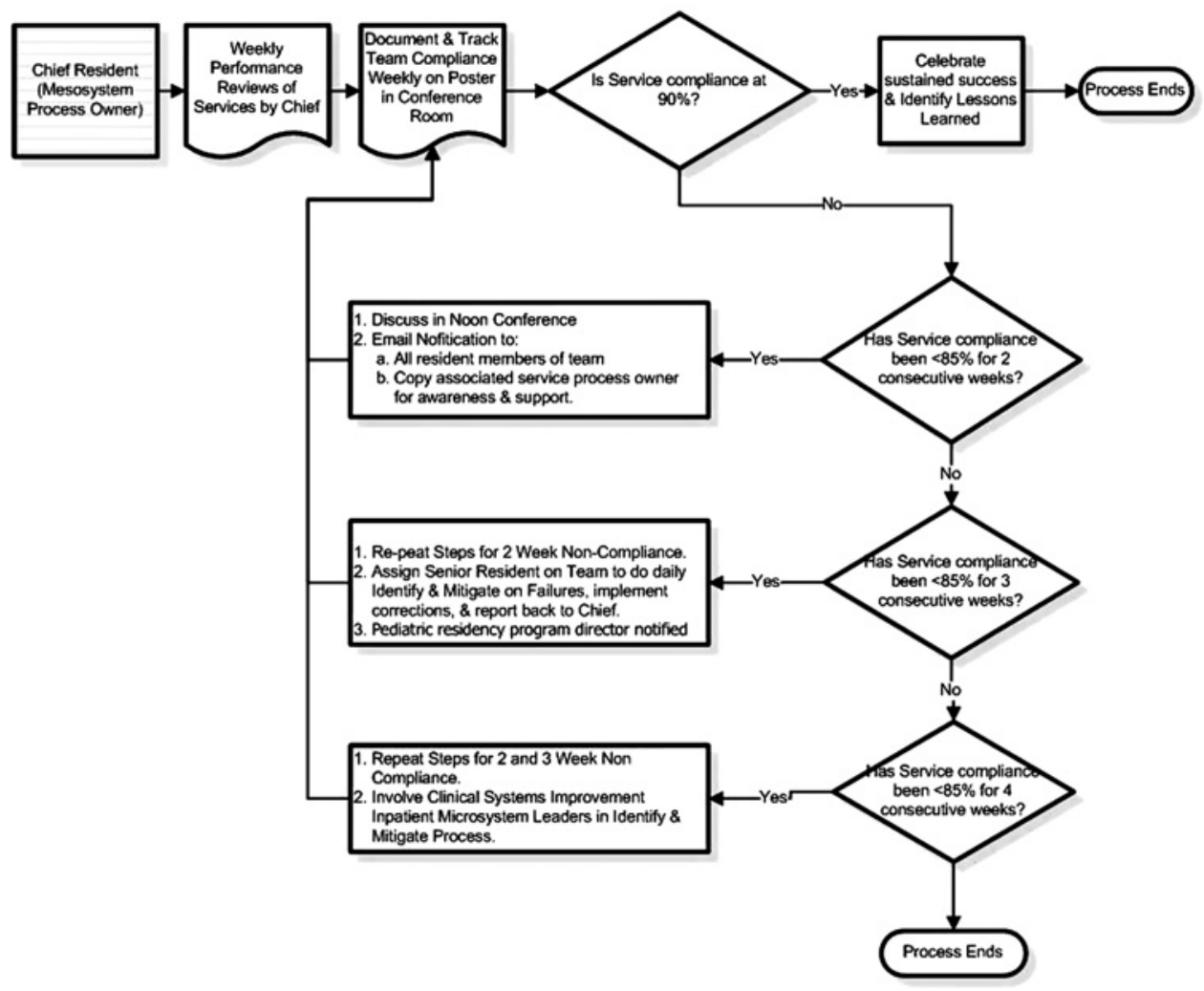


CCHMC Inpatient Medical Services Medication Reconciliation Weekly Performance Scorecard

\begin{tabular}{|c|c|c|c|c|c|c|c|c|c|c|c|c|c|c|c|c|c|c|c|c|c|c|c|c|c|c|c|}
\hline Service & & & & & & & & & & & & & & & & & & & & & & & & & & & \\
\hline $\begin{array}{c}\text { Adololesent/ Pulmonaryl } \\
\text { Nephrology }\end{array}$ & 100 & 100 & 100 & 100 & 100 & 100 & 92 & 100 & 94 & 100 & 87 & 92 & 100 & 100 & 100 & 86 & 100 & 100 & 82 & 100 & 86 & 100 & 100 & 100 & 100 & & \\
\hline Bone Marrow Transplant & 89 & 86 & 91 & 100 & 100 & 100 & 89 & 88 & 100 & 100 & 57 & 80 & 89 & 100 & 100 & 100 & 100 & 75 & 100 & 100 & 100 & 100 & \begin{tabular}{|l|}
75 \\
\end{tabular} & \begin{tabular}{|l|}
87 \\
\end{tabular} & 100 & & \\
\hline Cardiology & 88 & 75 & 75 & 100 & 100 & 100 & 91 & 83 & 100 & 73 & 86 & 88 & 78 & 100 & 67 & 86 & 100 & 91 & 100 & 40 & 86 & 50 & 100 & 93 & 100 & & \\
\hline Gastroenterology & 94 & 92 & 100 & 83 & 100 & 93 & 93 & 100 & 100 & 89 & 94 & 80 & 92 & 100 & 92 & 100 & 90 & 100 & 83 & 100 & 94 & 100 & 82 & 100 & 95 & & \\
\hline General Pediatrics & 97 & 97 & 98 & 96 & 99 & 97 & 95 & 99 & 95 & 97 & 97 & 98 & 98 & 97 & 92 & 98 & 97 & 97 & 93 & 95 & 94 & 98 & 99 & 97 & 100 & & \\
\hline Hematology & 100 & 86 & 91 & 100 & 86 & 80 & 73 & 83 & 100 & 100 & 89 & 88 & 100 & 93 & 100 & 100 & 100 & 100 & 93 & 100 & 100 & 100 & 100 & 100 & 80 & & \\
\hline Critical Care & 80 & 93 & 83 & 86 & 100 & 90 & 92 & 94 & 65 & 71 & 93 & 92 & 100 & 100 & 60 & 60 & 69 & 71 & 100 & 100 & 100 & 100 & 100 & 88 & 75 & & \\
\hline Oncology & 89 & 100 & 100 & 100 & 88 & 100 & 73 & 100 & 93 & 100 & 94 & 100 & 93 & 100 & 87 & 100 & 92 & 90 & 80 & 100 & 89 & 100 & 100 & 90 & 90 & & \\
\hline Neonatology & 100 & 67 & 93 & 89 & 100 & 9 & 100 & 100 & 94 & 80 & 77 & 79 & 100 & 83 & $\overline{95}$ & 84 & 75 & 76 & 88 & 89 & 100 & 88 & 85 & 88 & 93 & & \\
\hline Neurology & 100 & 100 & 100 & 93 & 100 & 100 & 100 & 100 & 100 & 95 & 93 & 94 & 100 & 100 & 95 & 100 & 100 & 100 & 100 & 100 & 100 & 100 & 100 & 95 & 100 & & \\
\hline Pulmonaryl Endocrine & 100 & 93 & 100 & 92 & 100 & 100 & 90 & 100 & 100 & 88 & 90 & 77 & 88 & 100 & 100 & 93 & 100 & 100 & 80 & 100 & 100 & 90 & 90 & 100 & 100 & & \\
\hline Rehabilitation & 100 & 100 & 100 & & & & 100 & & & 33 & & 100 & 100 & & & 100 & 100 & & & & 100 & 0 & 100 & & & & \\
\hline Rheumatology & 100 & 100 & 100 & & & 100 & \begin{tabular}{|l|}
67 \\
\end{tabular} & 100 & & 50 & 100 & \begin{tabular}{|l|}
100 \\
\end{tabular} & & 100 & 100 & & 100 & & 100 & 100 & \begin{tabular}{|c|}
100 \\
\end{tabular} & 100 & 100 & 50 & & & \\
\hline & 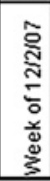 & 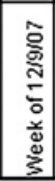 & 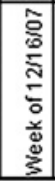 & 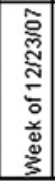 & 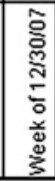 & 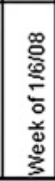 & 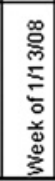 & 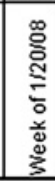 & 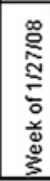 & 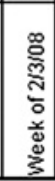 & 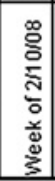 & 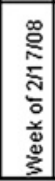 & 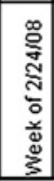 & 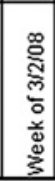 & 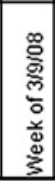 & 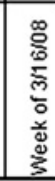 & 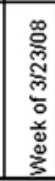 & 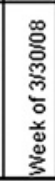 & 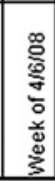 & 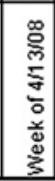 & 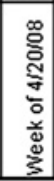 & 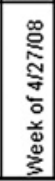 & 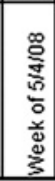 & 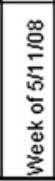 & 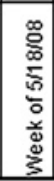 & 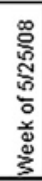 & 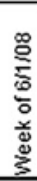 \\
\hline
\end{tabular}

Figure 6 Performance scorecard.

special-cause variation (variations not normally part of the system that appear due to specific events). ${ }^{12}$ Observed changes are considered special causes when any of the following criteria are true: there is a shift (eight or more consecutive points either above or below the mean/median centre line); there is a trend (six consecutive increasing or decreasing points); or there are alternating points (at least 14 consecutive points alternating above and below the mean/median centre line). A P control chart was constructed based on count data with a binomial distribution. ${ }^{13}$ The $\mathrm{P}$ chart considers data points derived from counts where the population sample size varies at each data point. The control limits take this variation in sample size into consideration, so the control limit lines do not appear as straight lines.

\section{Human subject protection}

The CCHMC institutional review board waived the requirement for written informed consent for this quality-improvement initiative, provided that no individuals were identified.

\section{RESULTS}

The number of medications per patient at admission for various age groups is shown in table 1. Approximately half of the children admitted were taking seven or more medications. For children less than 2 years of age, the percentage was even higher. The most common medications reconciled are shown in table 2. When the project was launched, the inpatient medical services were $62 \%$ compliant with completion of medical reconciliation within $24 \mathrm{~h}$ of admission. Within 9 months of implementation, medication reconciliation completion was $>90 \%$ hospital-wide. This was sustained at a mean of 93\% for 27 months (figure 7). At the unit level, critical care areas initially struggled the most with meeting medication reconciliation goals, but over time, they met expectations (figure 5).

\section{DISCUSSION}

We used reliability and improvement science methods to implement a successful and sustained medication reconciliation process. Improvement science is defined as applying the scientific method to making improvements to systems and processes that improve the delivery of healthcare. ${ }^{514}$ Improvement activities focused on the key drivers to successful and sustained medication reconciliation identified by the team following the failure mode and effects analysis: senior leadership support, simplification and standardisation of the reconciliation process and electronic application,

\begin{tabular}{|c|c|c|c|c|}
\hline $\begin{array}{l}\text { Patient age } \\
\text { (years) }\end{array}$ & $\begin{array}{l}\text { No }(\%) \text { of patients } \\
\text { with no medications } \\
(\mathrm{N}=22452)\end{array}$ & $\begin{array}{l}\text { No }(\%) \text { of patients } \\
\text { with } 1-2 \text { medications } \\
(\mathrm{N}=7830)\end{array}$ & $\begin{array}{l}\text { No }(\%) \text { of patients } \\
\text { with } 3-6 \text { medications } \\
(\mathrm{N}=6598)\end{array}$ & $\begin{array}{l}\text { No }(\%) \text { of patients } \\
\text { with } 7 \text { or more } \\
\text { medications }(\mathrm{N}=4305)\end{array}$ \\
\hline $0-2$ & $8674(20.1)$ & 2605 (9.5) & 1226 (3.4) & 446 (67.0) \\
\hline $2-8$ & $5762(16.1)$ & $2261(18.1)$ & $2171(18.5)$ & 1162 (47.3) \\
\hline $8-14$ & 3663 (19.9) & 1487 (19.1) & $1536(10.2)$ & 991 (50.7) \\
\hline$>14$ & 4353 (19.4) & 1477 (20.0) & 1665 (12.9) & $1706(47.7)$ \\
\hline
\end{tabular}


Table 2 Most common reconciled medications

\begin{tabular}{ll}
\hline Medication & No (\%) \\
\hline Albuterol & $4712(4.6)$ \\
Lansoprazole & $3448(3.4)$ \\
Miralax & $2173(2.1)$ \\
Acetaminophen & $1967(1.9)$ \\
Diazepam & $1894(1.9)$ \\
Ranitidine & $1706(1.7)$ \\
Ondansetron & $1516(1.5)$ \\
Montelukast & $1444(1.4)$ \\
Budesonide & $1329(1.3)$ \\
Fluticasone & $1258(1.2)$ \\
\hline
\end{tabular}

prescriber knowledge of expectations, and a highly reliable and visible system. We believe these elements are generalisable to other institutions.

Enhancements to the electronic application linking order entry to the medication reconciliation application, in particular, appeared to increase usability and reliability, leading to rapid improvement. Developed by industrial engineers, ${ }^{15}$ reliability science has been used effectively in complex industries such as nuclear power to improve both safety and the rate at which a system consistently produces appropriate outcomes. ${ }^{16-21}$ Reliability science has been applied to healthcare to help providers reduce defects in care or care processes, increase the consistency with which appropriate care is delivered and improve patient outcomes. ${ }^{22}$ We achieved $90 \%$ compliance when we had spread to seven of 13 medical services, accounting for $51 \%$ of inpatient admissions. The rotation of residents to other nonintervention services likely caused unintended spread of improvements prior to official implementation.

Our improvement team consisted of frontline physicians/residents, nurses and internal quality-improvement specialists. Initially, the chief residents were not included on our team. This was a mistake, as they knew the system and could hold residents accountable for medication reconciliation completion. Not until we engaged them were we able to make meaningful changes that drove progress. Having the right people on the team promoted improved communication and accountability that hardwired processes. ${ }^{23} 24$ Ensuring that frontline staff felt responsible for completing medication reconciliation (deference to expertise ${ }^{6}$ ) was instrumental to success. At initiation of the project, the team met weekly. As the project progressed, meetings occurred monthly. Not every site may have the personnel and resources to commit to this endeavour.

Most importantly, the use of improvement science methodology was instrumental in sustaining the process.

\section{Gle Cincinnati MChildren's}

\section{Medical Services \\ Inpatient Medication Reconciliation Compliance Upon Admission}

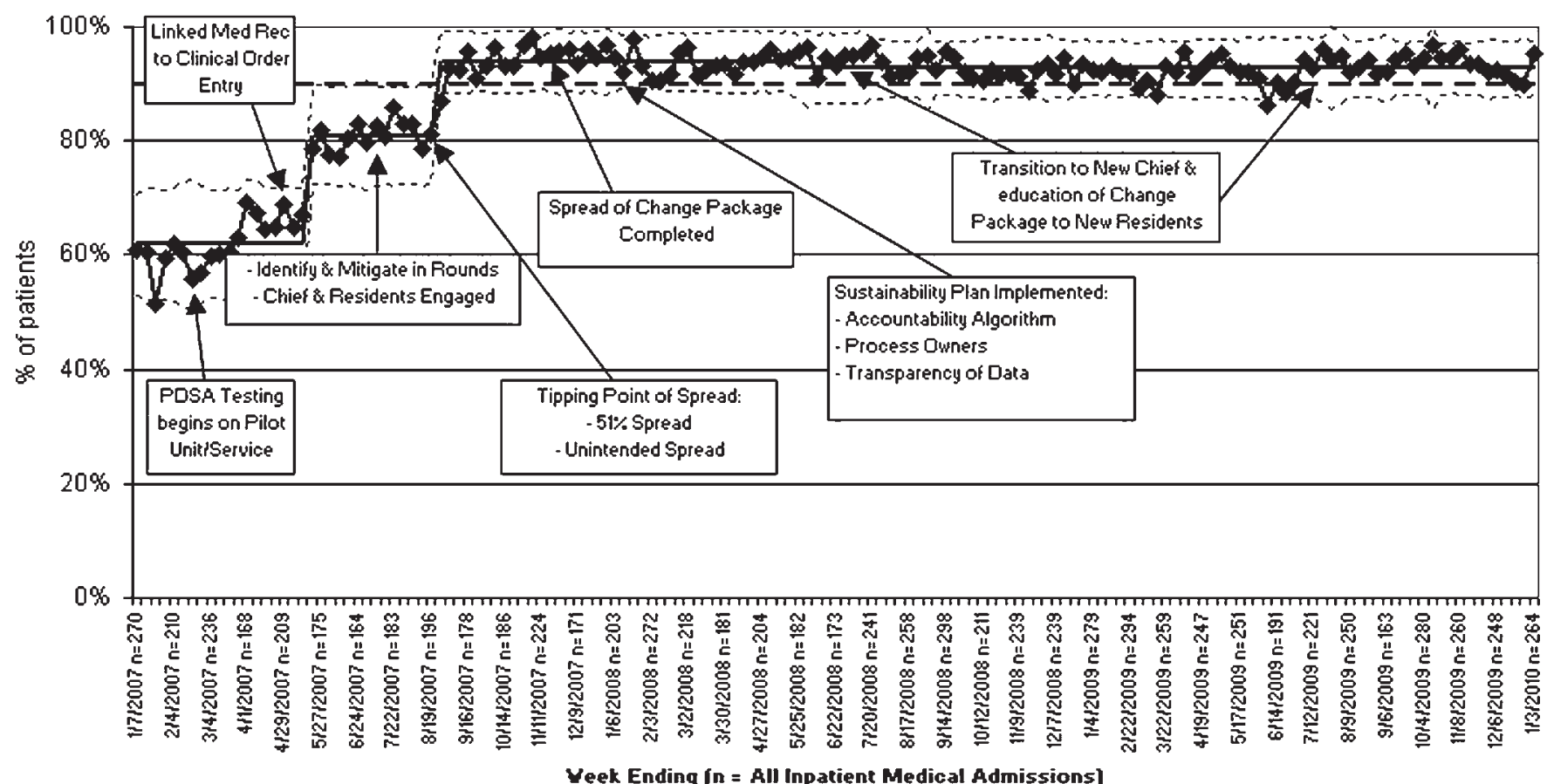

Yeek Ending (n = All Inpatient Medical Admissions)

$\longrightarrow$ \% Reconciled -Mean (\% Reconciled) -.... Upper Control Limit -...Lower Control Limit - - Goal (90\%)

Figure 7 Annotated P chart. PDSA, Plan-Do-Study-Act. 
With the key components of the sustainability plan in place (clear plan, accountability algorithm, process owners and transparent data), we have been able to sustain house-wide medication reconciliation compliance on inpatient medical services. Although this project was initially labour-intensive, once the team transitioned to a sustain model, a project manager spent only 45-60 min each week reviewing data and updating and submitting reports to unit and hospital leaders.

\section{Limitations}

In the testing phase, process measures were included in each of our PDSA cycles. Data collection on additional process measures was prohibited by cost and resources. We chose instead to focus resources on studying failures that had an impact on our main outcome measuremedication reconciliation completion. The availability of data on compliance rates within $48 \mathrm{~h}$ allowed us to quickly analyse and address failures.

There is strong evidence in the medical literature for adult patients linking medication reconciliation completion to reduction in ADEs, but evidence in paediatrics is lacking. ${ }^{25}{ }^{26}$ Initially, we assumed that most of our patients were on fewer high-risk medications than adults. Thus, we were surprised to find that many children on only one medication at admission were actually taking high-risk drugs, such as warfarin and filgrastim. Owing to the severe consequences of ADEs related to high-risk medications, our goal was to improve paediatric patients' medication reconciliation completion at admission. Although we were able to demonstrate improvement in reconciliation completion, we could not determine whether increased compliance led to a decrease in ADEs. Since the implementation of the reconciliation process, there has been an overall increase in reports of medical errors at CCHMC. This is believed to be a result of organisational-wide efforts to increase detection of patient harm while reducing our punitive culture. Currently, we do not have a reliable process to link reported medication errors to errors secondary to inaccurate medication reconciliation, so are unable to differentiate the underlying causes of reported ADEs. We are currently working on a dependable process to link errors in medication reconciliation to ADEs and to accurately capture medication reconciliation failures.

Although medication reconciliation was completed, accuracy remains a concern. A next step to improve the accuracy of medication history is to authenticate inpatient medication reconciliation with comprehensive pharmacy medication histories. Other hospitals have used pharmacists to perform medication reconciliation. ${ }^{27-30}$ Early PDSA cycle testing of reconciliation of high-risk medications by pharmacists at CCHMC actually prolonged completion by $15-50 \mathrm{~min}$ when they had to clarify orders with the prescribing physician. Our hospital did not have the personnel or resources for pharmacists to take on this role daily. As we implemented a new electronic medical record system, we integrated inpatient medication reconciliation with outpatient and pharmacy records to improve accuracy and incorporate medication reconciliation into medication management.

Also, the medication reconciliation prompt was not a 'hard stop' or an alert in an electronic medical record that did not allow one to proceed with orders or documentation until the required task (medication reconciliation) was performed. ${ }^{31}$ Thus, prescribers could bypass the system by clicking on the 'done' button when, in reality, medication reconciliation had not been completed. To address this concern, we built redundancy into the system. If a prescriber clicked the 'done' button and had not entered the medication reconciliation program, it was counted as a medication reconciliation failure. There is a possibility that a prescriber could enter the medication program and then hit the 'reconcile' button without truly reviewing the list and making changes. Unfortunately, we do not have data to determine how often this occurred. However, to help alleviate this concern, in our new electronic medical record, medication reconciliation is directly linked with placing inpatient orders and not a separate step.

\section{CONCLUSIONS}

We used quality-improvement methods and reliability science to successfully implement a sustainable process for medical reconciliation completion at admission at a large, busy academic children's hospital. In addition to performing well on this common quality measure, we believe this will increase the reliability of the care we deliver and may be a step in decreasing ADEs.

Acknowledgements The authors would like to thank A Carver, C Munda, R Mena, K Rich and the 2007-2008 chief residents for their help in completing this project.

Competing interests None.

Ethics approval Ethics approval was provided by the Cincinnati Children's Hospital Medical Center, Cincinnati, Ohio, USA.

Provenance and peer review Not commissioned; externally peer reviewed.

\section{REFERENCES}

1. Anon. Using medication reconciliation to prevent errors. Jt Comm J Qual Patient Saf 2006;32:230-2.

2. Massachusetts Coalition for the Prevention of Medical Errors. Reconciling medications: safe practice recommendations. http://www. macoalition.org/Initiatives/RecMeds/SafePractices.pdf (accessed 3 Aug 2009).

3. Clay BJ, Halasyamani L, Stucky ER, et al. Results of a medication reconciliation survey from the 2006 Society of Hospital Medicine national meeting. $J$ Hosp Med 2008;3:465-72.

4. Pedersen CA, Schneider PJ, Scheckelhoff DJ. ASHP national survey of pharmacy practice in hospital settings: monitoring and patient education-2009. Am J Health-Syst Pharm 2010;67:542-58. 
5. Langley GJ, Nolan KM, Nolan TW, et al. The Improvement Guide. A Practical Approach to Enhancing Organizational Performance. San Francisco: Jossey-Bass, 1996.

6. Luria JW, Muething SE, Schoettker PJ, et al. Reliability science and patient safety. Pediatr Clin North Am 2006;53:1121-33.

7. Cohen MR, Senders J, Davis NM. Failure mode and effects analysis: a novel approach to avoiding dangerous medication errors and accidents. Hosp Pharm 1994;29:319-30.

8. DeRosier J, Stalhandske E, Bagian JP, et al. Using health care Failure Mode and Effect Analysis: the VA National Center for Patient Safety's prospective risk analysis system. Jt Comm J Qual Improv 2002;28:248-67, 209.

9. Agency for Healthcare Research and Quality. Hospital Survey and Patient Safety Culture. http://www.ahrq.gov/qual/patientsafetyculture/ hospsurvindex.htm (accessed 22 Jun 2010).

10. Inamdar N, Kaplan RS, Bower M. Applying the balanced scorecard in healthcare provider organizations. J Healthc Manag 2002;47:179-95; discussion 195-6.

11. Wyatt J. Scorecards, dashboards, and KPIs keys to integrated performance measurement. Healthc Financ Manage 2004;58:76-80.

12. Moen RD, Nolan TW, Provost LP. Quality Improvement Through Planned Experimentation. 2nd edn. New York: McGraw-Hill, 1999.

13. Amin SG. Control charts 101: a guide to health care applications. Qual Manag Health Care 2001;9:1-27.

14. Deming WE. The New Economics for Industry, Government, Education. Cambridge, MA: Massachusetts Institute of Technology, Center for Advanced Engineering Study, 1993.

15. Ebeling CE. An Introduction to Reliability and Maintainability Engineering. New York: McGraw-Hill, 1997.

16. Weick KE. Organizational culture as a source of high reliability. Calif Manage Rev 1987;29:112-27.

17. Schulman PR. The negotiated order of organizational reliability. Adm Soc 1993;25:353-72.

18. Roberts $\mathrm{KH}$. Managing high reliability organizations. Calif Manage Rev 1990;32:101-13.
19. Roberts $\mathrm{KH}$. Some characteristics of one type of high reliability organization. Organ Sci 1990;1:160-76.

20. Bourrier M. Organizing maintenance work at two American nuclear power plants. J Contingencies Crisis Manage 1996;4:104-12.

21. Bierly PE, Spender JC. Culture and high reliability organizations: the case of the nuclear submarine. J Manag 1995;21:639-56.

22. Nolan T, Resar R, Haraden C, et al. Improving the Reliability of Health Care. Cambridge, MA: Institute for Healthcare Improvement, 2004. http://www.ihi.org/lHI/Products/WhitePapers/ ImprovingtheReliabilityofHealthCare.htm (accessed 9 Aug 2009).

23. Fortescue EB, Kaushal R, Landrigan CP, et al. Prioritizing strategies for preventing medication errors and averse drug events in pediatric inpatients. Pediatrics 2003;111:722-9.

24. Varkey P, Cunningham J, O'Meara J, et al. Multidisciplinary approach to inpatient medication reconciliation in an academic setting. Am J Health Syst Pharm 2007;64:850-4.

25. Haig K. Medication reconciliation. Am J Med Qual 2006;21:299-303.

26. Pronovost $\mathrm{P}$, Weast $\mathrm{B}$, Schwarz $\mathrm{M}$, et al. Medication reconciliation: a practical tool to reduce the risk of medication errors. $J$ Crit Care 2003:18:201-5.

27. Stone BL, Boehme S, Mundorff MB, et al. Hospital admission medication reconcilation in medically complex children: an observational study. Arch Dis Child 2010;95:250-5.

28. Murphy EM, Oxencis CJ, Klauck JA, et al. Medication reconcilation at an academic medical center: implementation of a comprehensive program from admission to discharge. Am J Health Syst Pharm 2009;66:2126-31.

29. Coffey M, Mack L, Streitenberger K, et al. Prevalence and clinical significance of medication discrepancies at pediatric hospital admission. Acad Pediatr 2009;9:360-5.e1.

30. Fertleman M, Barnett N, Patel T. Improving medication management for patients: the effect of a pharmacist on post-admission ward rounds. Qual Saf Health Care 2005;14:207-11.

31. Evans AS, Lazar EJ, Tiase VL, et al. The role of housestaff in implementing medication reconciliation on admission at an academic medical center. Am J Med Qual 2011;26:39-42. 